\title{
ON THE CAYLEY-HAMILTON PROPERTY IN ABELIAN GROUPS
}

\author{
ROBERT R. MILITELLO \\ Rhodes College, Department of Mathematics and Computer Science \\ 2000 North Parkway \\ Memphis, TN 38112 \\ Email: Militello@iRhodes \\ (Received September 25, 1991)
}

\begin{abstract}
In this paper, the work of Casacuberta and Hilton on the class of abelian $f g$-like groups is extended. These groups share much in common with the class of finitely generated abelian groups.
\end{abstract}

KEYWORDS. Localization, $f g$-like group, $f g p$ group.

1991 AMS SUBJECT CLASSIFICATION CODES. 20K40, $20 \mathrm{~K} 15$.

\section{INTRODUCTION}

A nilpotent group $G$ is said to be finitely generated at every prime $(f g p)$ if for each prime $p$ there is a finitely generated nilpotent group $M$ such that $G_{p} \simeq M_{p}$. If there exists a single finitely generated group $M$ which works for all primes, then we say $G$ is $f g$-like, or more specifically, $M$-like. Here $G_{p}$ denotes the $p$-localization of $G$. In this paper, we continue the study initiated by Casacuberta and Hilton on properties of $f g p$ groups. In particular, we focus our attention on annihilator properties, in the sense of the Cayley-Hamilton theorem, which an abelian group may satisfy.

Casacuberta and Hilton [2] showed that if $A$ is a special $f g p$ group, the following are equivalent:

(a) $A$ is $f g$-like;

(b) $\forall \phi: A \rightarrow A, \exists$ non-zero $F \in \mathbb{Z}[t]$ with $F(\phi)(A)=0$;

(c) $\forall \phi: A \rightarrow A, \exists$ monic $F \in \mathbb{Z}[t]$ with $F(\phi)(A)=0$;

(d) $\forall \phi: A \simeq A, \exists$ non-zero $F \in \mathbb{Z}[t]$ with $F(\phi)(A)=0$;

(e) $\forall \phi: A \simeq A, \exists$ demonic $F \in \mathbb{Z}[t]$ with $F(\phi)(A)=0$.

Here, a nilpotent group $G$ is called special if $T G \mapsto G \rightarrow F G$ splits on the right, where $T G$ is the torsion subgroup and $F G$ is the torsion-free quotient. For an abelian group $A$, this is equivalent to $A \simeq T A \oplus F A$. We should also mention that a demonic polynomial is a polynomial whose leading coefficient and constant coefficient are \pm 1 .

The theorem is proven by showing the following implications:

$$
\begin{array}{rlll}
(a) \Rightarrow \begin{array}{c}
(c) \\
\Downarrow
\end{array} & \Rightarrow(\epsilon) \\
& \Downarrow \\
& (b) \Rightarrow(d) \Rightarrow(a)
\end{array}
$$

Only the implication $(d) \Rightarrow(a)$ requires that $A$ be special. Naturally, one should ask whether 
there exist $f g p$ groups which are non-special (and hence are not $f g$-like) which satisfy property (d). Casacuberta and Hilton have shown the existence of such a group. In this paper, we show that there does exist a large class of $f g p$ groups which are non-special and which do not satisfy property $(d)$.

We will say that an abelian group $A$ is Cayley-Hamilton if it satisfies property (c). We will call $A$ almost Cayley-Hamilton if it satisfies property $(d)$. Obviously, Cayley-Hamilton implies almost Cayley-Hamilton; however, it is easy to see that the converse is not true. Thus, let $A=\bigoplus_{\imath=-\infty}^{\infty} \mathbb{Z} / 2$. Then $F(t)=2 t$ annihilates $\phi$ for any $\phi: A \rightarrow A$. However, if we let the $i^{t h}$ copy of $\mathbb{Z} / 2$ be generated by $x_{\imath}$, then $\phi: A \simeq A$, given by $\phi\left(x_{\imath}\right)=x_{\imath+1}$, cannot be annihilated by any polynomial which is not a multiple of 2 .

In Section 2, our goal is to show that if $A$ is an abelian group whose torsion-free quotient is $\mathbf{Z}$-like and if, for an infinite number of primes $p, T A_{p}$ contains either $\mathbf{Z} / p^{n(p)} \oplus \mathbf{Z} / p^{n(p)}$ or $\mathbb{Z} / p^{n(p)} \oplus \mathbb{Z} / p^{n(p)+1}$ as a direct summand, then $A$ is not almost Cayley-Hamilton. In Section 3, we generalize the results of Section 2 by replacing the condition that the torsion-free quotient is $\mathbf{Z}$ like by the condition that the torsion-free quotient is $\mathbf{Z}^{k}$-like. Of course it is natural to conjecture that the theorem of Casacuberta and Hilton remains true if one considers any fgp groups which are special or have, for an infinite number of primes, non-cyclic torsion components. One should note that our results do not require that $A$ is $f g p$. We only assume that the torsion-free quotient is $\mathbb{Z}^{k}$-like. We call such groups almost $f g p$ and we describe some properties of these groups in Section 3.

In a forthcoming paper, the author will discuss a generalization of the Cayley-Hamilton property in the class of solvable groups.

This paper is based on research that formed part of the author's dissertation at SUNY Binghamton. Special thanks to Professor Peter Hilton for advising the dissertation research.

\section{ON ALMOST $f g p$ ABELIAN GROUPS OF RANK ONE.}

Let $A$ be an abelian group. We denote by [A] the class of the extension $T A \mapsto A \rightarrow F A$ in the abelian group $\operatorname{Ext}(F A, T A)$. We will sometimes write $T$ for $T A$ and $F$ for $F A$.

Initially, we will place no restrictions on $T=T A$. However, we will assume throughout that $F=F A$ is $\mathbb{Z}^{k}$-like for some positive integer $k$. We will refer to such abelian groups as being almost $f g p$. It is clear that an almost $f g p$ group is $f g p$ if and only if $T_{p}=T A_{p}$ is finite for each prime $p$.

Our first objective is to compute $\operatorname{Ext}(F, T)$ when $k=1$. To do this, we identify $F$ with a group of pseudo-integers (see [5]). Explicitly, let $F=\left\langle\frac{1}{p^{k(p)}}\right|$ all primes $p$, where $k(p)$ is a non-negative integer for each prime $p\rangle \subseteq \mathbb{Q}$.

LEMMA 1. If $F$ is as above and $T$ is torsion, then $\operatorname{Ext}(F, T)=\frac{\prod_{p} T_{p} / p^{k(p)} T_{p}}{\underset{p}{\oplus} T_{p} / p^{k(p)} T_{p}}$.

PROOF. Consider the exact sequence $\mathbb{Z} \mapsto F \rightarrow F / \mathbb{Z}=\underset{p}{\oplus} \mathbf{Z} / p^{k(p)}$.

This sequence induces the following right-exact sequence:

$$
\operatorname{Hom}(\mathbb{Z}, T) \stackrel{\Delta}{\rightarrow} \operatorname{Ext}\left(\underset{p}{\oplus \mathbb{Z} / p^{k(p)}}, T\right) \rightarrow \operatorname{Ext}(F, T) .
$$


However, $\left.\operatorname{Ext} \underset{p}{\oplus \mathbf{Z}} / p^{k(p)}, T\right)=\prod_{p} \operatorname{Ext}\left(\mathbf{Z} / p^{k(p)}, T\right)=\prod_{p} T / p^{k(p)} T=\prod_{p} T_{p} / p^{k(p)} T_{p}$ Further, $\operatorname{Hom}(\mathbf{Z}, T)=$ $T=\underset{p}{\oplus} T_{p}$ and $\Delta: \underset{p}{\oplus} T_{p} \rightarrow \prod_{p} T_{p} / p^{k(p)} T_{p}$ is the canonical map. Hence $\Delta(T)=\underset{p}{\oplus} T_{p} / p^{k(p)} T_{p}$. Since the sequence is right-exact, it follows that $\operatorname{Ext}(F, T)=\frac{\prod_{p} T_{p} / p^{k(p)} T_{p}}{\underset{p}{\oplus T_{p} / p^{k(p)} T_{p}}}$.

Q.E.D.

Obviously, if $T$ is almost torsion-free, then $\operatorname{Ext}(F, T)=0$. Hence, $A=T \oplus F$ and $A$ is special. Of course this result follows from a theorem of Casacuberta and Hilton [1] which states that if $A$ is $B$-like and $T$ is almost torsion-free, then $\operatorname{Ext}(A, T) \simeq \operatorname{Ext}(B, T)$. We formally state this result as:

THEOREM 2. An almost $f g p$ abelian group of rank one which is almost torsion-free is special.

An application of Lémma 1.5 in Casacuberta and Hilton [2], gives the following corollary:

COROLLARY 3. An almost $f g p$ abelian group of rank one which is almost torsion-free is Cayley-Hamilton (almost Cayley-Hamilton) if and only if $T_{p}$ is Cayley-Hamilton (almost CayleyHamilton) for each prime $p$.

We now explore property $(d)$ in the class of almost $f g p$ groups of rank one which are not almost torsion-free. We will write $\left[\left(\bar{w}_{p}\right)\right]$ for $[A]$ where $w_{p} \in T_{p}$, and $\bar{w}_{p}$ is the coset of $w_{p}$ modulo $p^{k(p)} T_{p}$. The next result serves as a prelude for the main theorem of this section.

THEOREM 4. Let $A$ be an abelian group and let $F$ be $\mathbf{Z}$-like. Suppose that, for infinitely many primes $p, T_{p} \neq 0$ and $\bar{w}_{p}=0$. Then $A$ is not almost Cayley-Hamilton.

PROOF. We adapt an argument of Casacuberta and Hilton in [2]. Let $p_{1}, p_{2}, \ldots$ be an enumeration of the primes with the stated property. Let $\lambda_{1}, \lambda_{2}, \ldots$ be a sequence of positive integers such that each positive integer occurs infinitely often. As in [2], set $\mu_{2}=\left\{\begin{array}{ll}1 & \text { if } p_{2} \mid \lambda_{2} \\ \lambda_{2} & \text { if } p_{2} \nmid \lambda_{2}\end{array}\right.$ and define $\phi_{p_{\mathrm{t}}}^{\prime}: T_{p_{\mathrm{t}}} \rightarrow T_{p_{\mathrm{t}}}$ by $\phi_{p_{\mathrm{t}}}^{\prime}(x)=\mu_{\mathrm{z}} x$. If $q$ is a prime not included in the above enumeration, let $\phi_{q}^{\prime}=\mathrm{id}: T_{q} \rightarrow T_{q}$. Let $\phi^{\prime}=\sum_{p} \phi_{p}^{\prime}: T \rightarrow T$. Note that $\phi^{\prime}$ is an automorphism of $T$. Since $\bar{w}_{p}=0$ for $p=p_{1}, p_{2}, \ldots$ and $\phi_{q}^{\prime}=$ id for $q \neq p_{1}, p_{2}, \ldots$ it follows that $\phi_{*}^{\prime}[A]=[A]$. Thus $\phi^{\prime}$ extends to an endomorphism $\phi$ of $A$ which induces the identity on $F$. Thus $\phi$ is an automorphism of $A$. Now let $G$ be a non-zero polynomial over $\mathbf{Z}$ with $G(\phi)(A)=0$. Then $G\left(\phi^{\prime}\right)(T)=0$. Let $n$ be a positive integer. Note that $n=\mu_{2}$ for infinitely many $i$, say $j_{1}, j_{2}, \ldots$. For any given $j$ in the previous sequence, let $x_{j} \in T_{p,}-0$. Then $0=G\left(\phi^{\prime}\right)\left(x_{j}\right)=G\left(\mu_{j}\right)\left(x_{j}\right)$. Since $x_{j} \neq 0$, it follows that $p_{j} \mid G\left(\mu_{3}\right)$. Hence for $j=j_{1}, j_{2}, \ldots$ it follows that $p, \mid G(n)$. Hence $G(n)=0$. It follows that $G \equiv 0$ and $A$ is not almost Cayley-Hamilton.

Q.E.D.

COROLLARY 5. Let $A$ be an abelian group and let $F$ be $\mathbf{Z}$-like. Suppose that, for infinitely many primes $p, T_{p} \neq 0$ while $k(p)=0$. Then $A$ is not almost Cayley-Hamilton.

PROOF. For the infinite number of primes specified, $\bar{w}_{p}=0$. Now apply Theorem 4 . 
We now place conditions on $T$ in order to prove our main result and its corollary.

THEOREM 6. Suppose that for infinitely many $p, T_{p}$ is not cyclic, and $k(p) \neq 0$. Call this set of primes $S$. For each $p \in S$, write $T_{p}=\mathbb{Z} / p^{m(p)} \oplus \mathbb{Z} / p^{n(p)} \oplus$ (finite abelian $p$-group), where. for each $p, m(p)$ and $n(p)$ are positive integers with $n(p) \geq m(p)$. Let $\left\langle x_{p}\right\rangle=\mathbf{Z} / p^{m(p)}$ and $\left\langle y_{p}\right\rangle=\mathbb{Z} / p^{n(p)}$ and write $w_{p}=\left(r_{p} x_{p}, q_{p} y_{p}, \ldots\right)$ for some integers $r_{p}$ and $q_{p}$ which depend on $p$. If any one of the following conditions hold, then $A$ is not almost Cayley-Hamilton.

(a) $q_{p}$ or $r_{p}$ may be chosen to equal 0 for infinitely many $p \in S$.

(b) $q_{p}, r_{p}$ may be chosen so that $\left|q_{p}\right|_{p} \leq\left|r_{p}\right|_{p}$ for infinitely many $p \in S$.

(c) $q_{p}, r_{p}$ may be chosen so that $\left|r_{p}\right|_{p}+n(p) \leq\left|q_{p}\right|_{p}+m(p)$ for infinitely many $p \in S$.

Here $|t|_{p}$ is the exact power of $p$ which divides $t$.

PROOF. If either $q_{p}=0$ or $r_{p}=0$ for infinitely many $p \in S$, then a generalization of the argument in Theorem 4 shows that $A$ is not almost Cayley-Hamilton.

Suppose that $(b)$ holds. Let $S^{\prime} \subseteq S$ be the set of primes mentioned in $(b)$. Let $p_{1}, p_{2}, \ldots$, be an enumeration of the primes in $S^{\prime}$. Let $\lambda_{1}, \lambda_{2}, \ldots$ be a sequence of positive integers such that each positive integer appears infinitely often. Let $\mu_{p_{i}}=\left\{\begin{array}{ll}1 & \text { if } p_{2} \mid \lambda_{2} \\ \lambda_{i} & \text { if } p_{2} \nmid \lambda_{2}\end{array}\right.$. For each prime $p$ in $S^{\prime}$, define $\psi_{p}: \mathbb{Z} / p^{n(p)} \rightarrow \mathbb{Z} / p^{m(p)}$ so that $q_{p} \psi_{p}\left(y_{p}\right)=r_{p}\left(1-\mu_{p}\right) x_{p}$. Condition $(b)$ and the fact that $n(p) \geq m(p)$ guarantee that this is possible. Let $M_{p}=\left[\begin{array}{cc}\mu_{p} & \psi_{p} \\ 0 & 1\end{array}\right]$ and let $\phi_{p}^{\prime}: T_{p} \rightarrow T_{p}$ be defined by the matrix $\left[\begin{array}{cc}M_{p} & 0 \\ 0 & I\end{array}\right]$. Again, it is easy to see that $\phi_{p}^{\prime}$ is an automorphism of $T_{p}$. For $p \notin S^{\prime}$, let $\phi_{p}^{\prime}=$ id. Let $\phi^{\prime}=\sum_{p} \phi_{p}^{\prime}: T \rightarrow T$. We observe that $\phi^{\prime}$ is an automorphism of $T$. The formula $q_{p} \psi_{p}\left(y_{p}\right)=r_{p}\left(1-\mu_{p}\right) x_{p}$ guarantees $\phi_{p}^{\prime} w_{p}=w_{p}$. Hence $\phi_{*}^{\prime}[A]=[A]$. Thus $\phi^{\prime}$ extends to an automorphism $\phi: A \rightarrow A$ which induces the identity on $F$.

Finally, note that $\phi_{p}^{\prime}\left(x_{p}\right)=\mu_{p} x_{p}$. As in the proof of Theorem 4, there does not exist a nonzero polynomial over $\mathbb{Z}$ which annihilates $\phi^{\prime}$. Hence there does not exist such a polynomial which annihilates $\phi$. Hence $A$ is not almost Cayley-Hamilton. The proof using condition $(c)$ is similar and depends on defining a homomorphism $\psi_{p}^{\prime}: \mathbb{Z} / p^{m(p)} \rightarrow \mathbb{Z} / p^{n(p)}$ with properties similar to those of $\phi_{p}^{\prime}$ above.

COROLLARY 7. Suppose that, for infinitely many $p, T_{p}$ contains either $\mathbb{Z} / p^{n(p)} \oplus \mathbb{Z} / p^{n(p)}$ or $\mathbb{Z} / p^{n(p)} \oplus \mathbb{Z} / p^{n(p)+1}$ as a direct summand. Then $A$ is not almost Cayley-Hamilton.

Now let $T_{p}=\mathbb{Z} / p=\left\langle w_{p}\right\rangle$ for each prime $p$. Let $F=\left\langle\frac{1}{p}\right|$ all primes $\left.p\right\rangle$ and let $A$ be the abelian group defined by $[A]=\left[\left(w_{p}\right)\right] \in \operatorname{Ext}(F, T)$. It was shown by Casacuberta and Hilton [2] that $A$ is Cayley-Hamilton. The following lemma shows that $A \otimes A$ satisfies the hypothesis of Corollary 7. Thus, the tensor product of Cayley-Hamilton groups need not be almost Cayley-Hamilton.

LEMMA 8. Let $A$ and $B$ be almost $f g p$ groups where $F A$ is $\mathbb{Z}^{k}$-like and $F B$ is $\mathbb{Z}^{l}$-like. Then $A \otimes B$ is almost $f g p$ where $F(A \otimes B)$ is $\mathbb{Z}^{k l}$-like. Moreover, $T(A \otimes B)_{p}=T A_{p}^{l} \oplus T B_{p}^{k} \oplus\left(T A_{p} \otimes T B_{p}\right)$.

PROOF. Note that $A_{p}=\mathbb{Z}_{p}^{k} \oplus T A_{p}$ and $B_{p}=\mathbb{Z}_{p}^{l} \oplus T B_{p}$. Then $(A \otimes B)_{p}=A_{p} \otimes B_{p}=$

$$
\begin{aligned}
\left(\mathbb{Z}_{p}^{k} \oplus T A_{p}\right) \otimes\left(\mathbb{Z}_{p}^{l} \oplus T B_{p}\right) & =\left(\mathbb{Z}_{p}^{k} \otimes \mathbb{Z}_{p}^{l}\right) \oplus\left(T A_{p} \otimes \mathbb{Z}_{p}^{l}\right) \oplus\left(\mathbb{Z}_{p}^{k} \otimes T B_{p}\right) \oplus\left(T A_{p} \otimes T B_{p}\right) . \\
& =\mathbb{Z}_{p}^{k l} \oplus T A_{p}^{l} \oplus T B_{p}^{k} \oplus\left(T A_{p} \otimes T B_{p}\right) .
\end{aligned}
$$


The result follows since $T(A \otimes B)_{p}=T\left((A \otimes B)_{p}\right)$ and $F(A \otimes B)_{p}=F\left((A \otimes B)_{p}\right)$.

An amusing consequence of Lemma 8 is that if $A$ and $B$ are almost $f g p$ and neither $A$ or $B$ is torsion, then, if $A \otimes B$ is $f g$-like, it follows that $A$ and $B$ are $f g$-like.

\section{ON ALMOST $f g p$ ABELIAN GROUPS.}

We now seek to generalize the results of Section 2. Given a $\mathbf{Z}^{k}$-like group $F$, we choose a reduced representation $R_{*}$ for $F$ in the sense of Casacuberta and Hilton [1]. Then we may replace $F$ by the subgroup of $\mathbb{Q}^{k}$ generated by the column vectors of $R_{p}$ for each prime $p$. Casacuberta and Hilton have shown [1] that $F$ contains $\mathbf{Z}^{k}$. The quotient $\bar{F}=F / \mathbf{Z}^{k}$ is a torsion group. Moreover, $\bar{F}_{p}$ is generated sby the cosets containing the column vectors of $R_{p}$.

EXAMPLE 9.. Let $R_{p}=\left[\begin{array}{cc}1 / p & 1 \\ 0 & 1 / p\end{array}\right]$. Then $\bar{F}_{p}=\left\langle\left(1, \frac{1}{p}\right)+\mathbf{Z}^{2},\left(\frac{1}{p}, 0\right)+\mathbf{Z}^{2}\right\rangle \simeq \mathbf{Z} / p \oplus \mathbf{Z} / p$.

EXAMPLE 10. Let $R_{p}=\left[\begin{array}{cc}1 / p & 1 / p^{2} \\ 0 & 1\end{array}\right]$. Then $\bar{F}_{p}=\left\langle\left(\frac{1}{p^{2}}, 1\right)+\mathbf{Z}^{2}\right\rangle \simeq \mathbf{Z} / p^{2}$.

In general, we may write $\bar{F}_{p} \simeq \mathbb{Z} / p^{n_{1}(p)} \oplus \mathbf{Z} / p^{n_{2}(p)} \oplus \cdots \oplus \mathbf{Z} / p^{n_{k}(p)}$ where $n_{1}(p) \leq n_{2}(p) \leq$ $\cdots \leq n_{k}(p)$ and some of the factors may be trivial.

LEMMA 11. If $T$ is torsion and $F$ is $\mathbf{Z}^{k}$-like, then

$$
\operatorname{Ext}(F, T)=\frac{\prod_{p}\left[T_{p} / p^{n_{1}(p)} T_{p} \oplus \cdots \oplus T_{p} / p^{n_{k}(p)} T_{p}\right]}{\oplus_{p}^{\oplus}\left[T_{p} / p^{n_{1}(p)} T_{p} \oplus \cdots \oplus T_{p} / p^{n_{k}(p)} T_{p}\right]}
$$

PROOF.. Consider the exact sequence $\mathbf{Z}^{k} \mapsto F \rightarrow \bar{F}=\underset{p}{\oplus} \bar{F}_{p}$.

This sequence induces the following right-exact sequence:

However,

$$
\left.\operatorname{Hom}\left(\mathbf{Z}^{k}, T\right) \stackrel{\Delta}{\rightarrow} \operatorname{Ext} \underset{p}{\oplus} \bar{F}_{p}, T\right) \rightarrow \operatorname{Ext}(F, T) .
$$

$$
\begin{aligned}
\operatorname{Ext}\left(\underset{p}{\oplus} \bar{F}_{p}, T\right)=\prod_{p} \operatorname{Ext}\left(\bar{F}_{p}, T\right) & =\prod_{p} \operatorname{Ext}\left(\mathbf{Z} / p^{n_{1}(p)} \oplus \cdots \oplus \mathbf{Z} / p^{n_{k}(p)}, T\right) \\
& =\prod_{p}\left[\operatorname{Ext}\left(\mathbf{Z} / p^{n_{1}(p)}, T\right) \oplus \cdots \oplus \operatorname{Ext}\left(\mathbf{Z} / p^{n_{k}(p)}, T\right)\right] . \\
& =\prod_{p}\left[T / p^{n_{1}(p)} T \oplus \cdots \oplus T / p^{n_{k}(p)} T\right] \\
& =\prod_{p}\left[T_{p} / p^{n_{1}(p) T_{p}} \oplus \cdots \oplus T_{p} / p^{n_{k}(p)} T_{p}\right] .
\end{aligned}
$$

Also $\operatorname{Hom}\left(\mathbf{Z}^{k}, T\right)=T^{k}=\left(\underset{p}{\oplus} T_{p}\right)^{k}$ and $\left.\Delta: \underset{p}{\oplus} T_{p}\right)^{k} \rightarrow \prod_{p}\left[T_{p} / p^{n_{1}(p)} T_{p} \oplus \cdots \oplus \oplus T_{p} / p^{n_{k}(p)} T_{p}\right]$ is the canonical map.

Thus $\Delta\left(\left(\underset{p}{\oplus} T_{p}\right)^{k}\right)=\underset{p}{\oplus}\left[T_{p} / p^{n_{1}(p) T_{p}} \oplus \cdots \oplus T_{p} / p^{n_{k}(p)} T_{p}\right]$. Since the above sequence is right exact, the result follows.

Q.E.D.

Once again, it is clear from Lemma 11 that if $T$ is almost torsion-free, then $A$ is special. Hence we have the following generalizations of Theorem 2 and Corollary 3. 
THEOREM 12.. An almost $f g p$, almost torsion-free group is special.

COROLLARY 13.. An almost $f g p$, almost torsion-free group is Cayley-Hamilton (almost Cayley-Hamilton) if and only if $T_{p}$ is Cayley-Hanilton (almost Cayley-Hamilton) for each prime $p$.

We also obtain generalizations of Theorem 4 and Corollary 5.

THEOREM 14 .. Suppose that, for infinitely many primes $p, w_{p}=0$ while $T_{p} \neq 0$. Then $A$ is not almost Cayley-Hamilton.

COROLLARY 15.. Suppose that, for infinitely many primes $p, n_{1}(p) \geq \log _{p} \exp \left(T_{p}\right)$ and $T_{p} \neq 0$. Then $A$ is not almost Cayley-Hamilton.

Now we seek to extend Corollary 7. Before doing this we need to place an additional hypothesis on $F$.

DEFINITION 16. Let $R_{*}$ be a reduced representation for $F$. $R_{*}$ is called collapsible if for each prime $p, \bar{F}_{p}$ is cyclic. In this case, $\bar{F}_{p} \simeq \mathbf{Z} / p^{n_{k}(p)}$. If $F$ is an arbitrary $\mathbf{Z}^{k}$-like group, then we call $F$ collapsible if there exists a reduced representation of $F$ which is collapsible.

Note that all $\mathbf{Z}$-like groups are collapsible. As a result, the following theorem generalizes Corollary 7.

THEOREM 17. Suppose that $F$ is $\mathbf{Z}^{k}$-like for $k \geq 1$ and that $F$ is collapsible. Suppose that, for an infinite number of primes $p, T_{p}$ contains either $\mathbf{Z} / p^{n(p)} \oplus \mathbf{Z} / p^{n(p)}$ or $\mathbf{Z} / p^{n(p)} \oplus \mathbf{Z} / p^{n(p)+1}$ as a direct summand. Then $A$ is not almost Cayley-Hamilton.

We close by mentioning that the class of almost $f g p$ abelian groups is a somewhat well behaved class of abelian groups. It is straight forward to show that the class is closed under the formation of quotients, subgroups, tensor products, torsion products, and homology. However, in contrast to the class of $f g p$ abelian groups, this class is not a Serre class because it fails to satisfy the condition of closure under extensions. For example, in the exact sequence $\mathbf{Z} \mapsto \mathbf{Q} \rightarrow \mathbf{Q} / \mathbf{Z}$ it is clear that $\mathbf{Z}$ and $Q / Z$ are almost $f g p$; however, $Q$ is clearly not almost $f g p$. We are presently exploring properties of nilpotent groups whose torsion-free quotient is $f g$-like.

\section{REFERENCES}

1. CASACUBERTA, J. and HILTON, P., On the extended genus of finitely generated abelian groups, Bull. Soc. Math. Belg., Sér A, 41, n.1 (1989), 51-72.

2. CASACUBERTA, J. and HILTON, P., On special nilpotent groups, Bull. Soc. Math. Belg., Sér B, 41, n.9 (1989), 261-273.

3. CASACUBERTA, J. and HILTON, P. , On nilpotent groups which are finitely generated at every prime, (to appear in Expositiones Mathematicae).

4. HILTON, P., On all sorts of automorphisms Amer. Math. Monthly 92 (1985), 650-656.

5. HILTON, P., On groups of pseudo-integers, Acta Math. Sinica (new series) 4, no. 2 (1988), 189-192.

6. HILTON, P., On a Serre class of nilpotent groups, (to appear in the Journal of Pure and Applied Algebra).

7. HILTON, P., MISLIN, G., ROITBERG, J., Localization of Nilpotent Groups and Spaces, North-Holland Math. Studies, 15, (1975). 


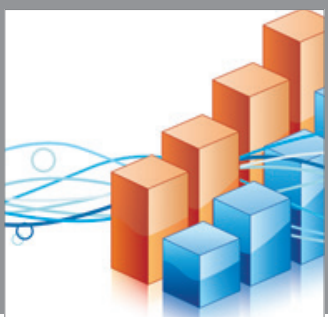

Advances in

Operations Research

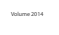

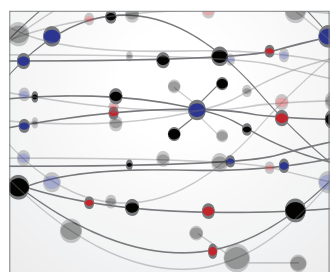

\section{The Scientific} World Journal
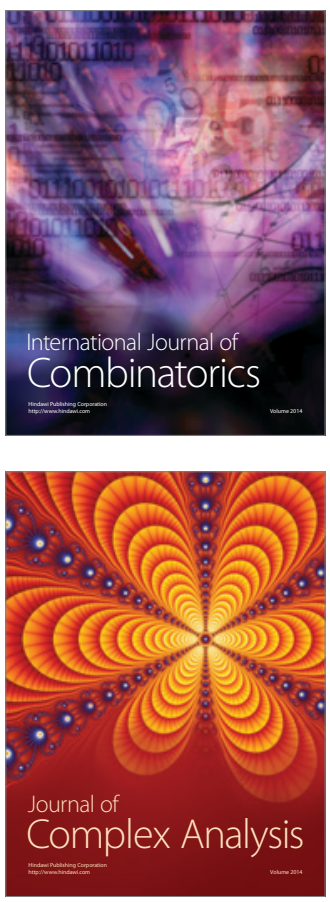

International Journal of

Mathematics and

Mathematical

Sciences
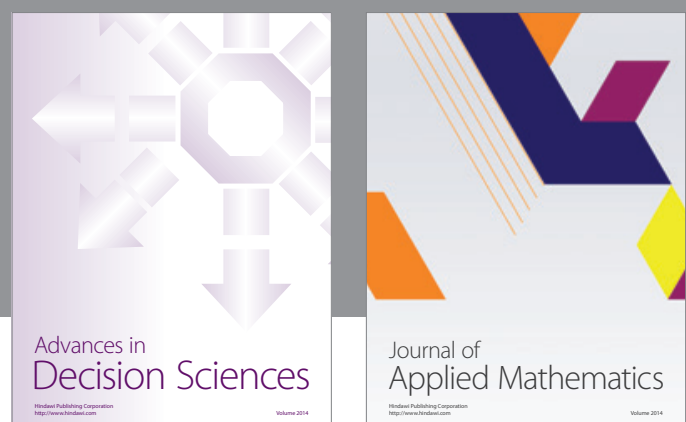

Journal of

Applied Mathematics
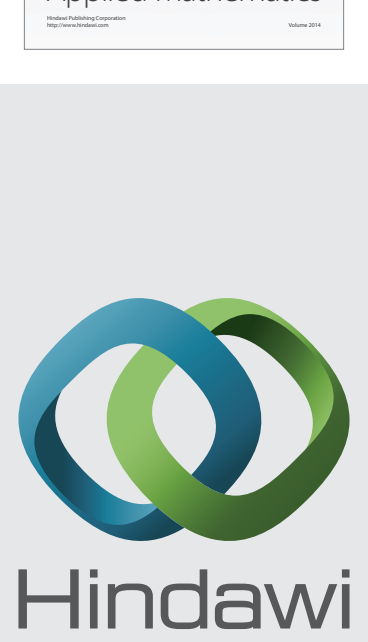

Submit your manuscripts at http://www.hindawi.com
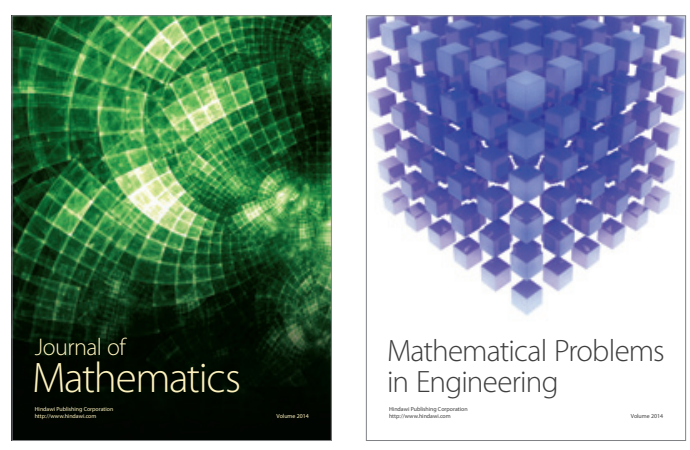

Mathematical Problems in Engineering
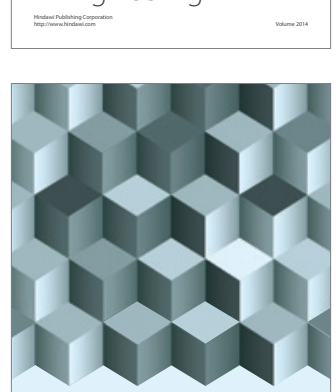

Journal of

Function Spaces
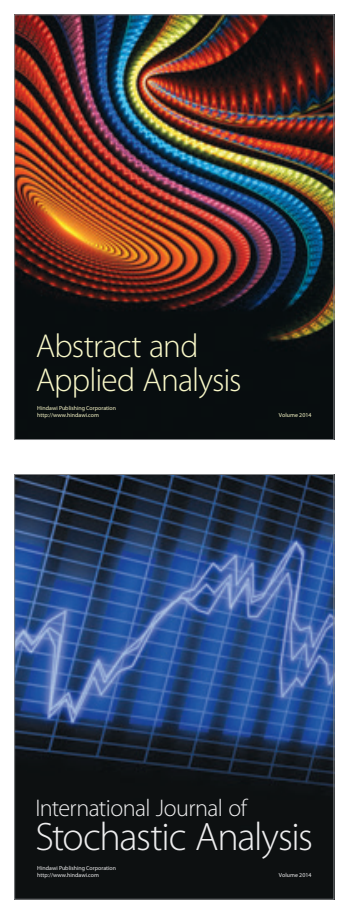

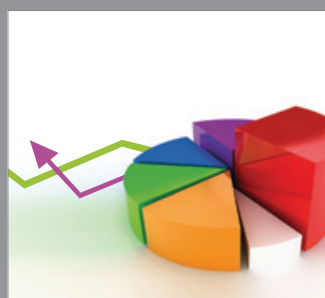

ournal of

Probability and Statistics

Promensencen
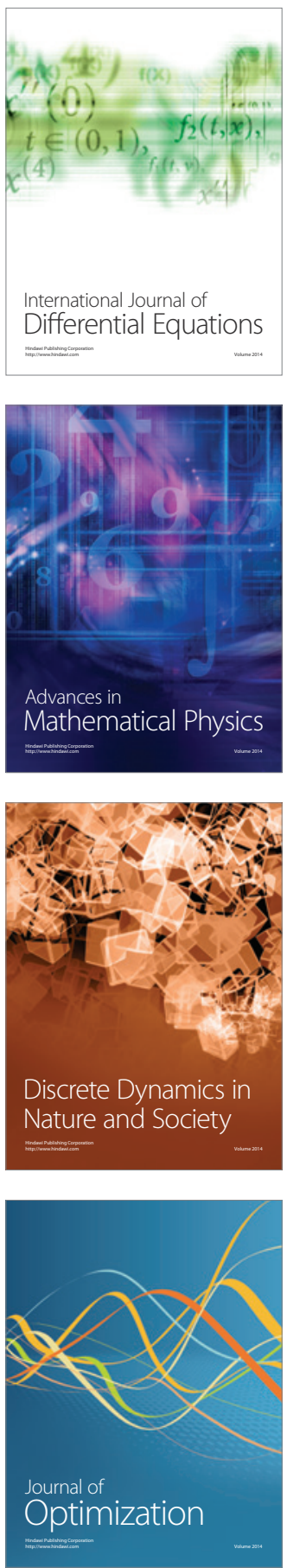